THURSDAY, JANUARY 25,1872

\section{THE SOLAR ECLIPSE}

\section{ACCOUNT OF OBSERVATIONS MADE AT POODOCOTTAH}

7 HE spectral observations of recent total eclipses of the sun had plainly demonstrated the existence of an incandescent gaseous stratum or atmosphere, surmounting the chromosphere or stratum of hydrogen which envelops the body of the sun, but they had not sufficed to determine its true conformation and extent. This question, therefore, constituted one of the principal problems remaining to be solved by observations of the eclipse of the izth of December, 1871 .

The slit-spectroscope applied to large telescopes doubtless affords the best means of verifying the existence, in the circumsolar regions, of this gaseous stratum, which may be termed the superior chromosphere, and of determining the materials of which it is composed; but from the shortness of the time available in an eclipse, the spectroscope can furnish only partial and local results, insufficient, therefore, to reveal the true structure, form, and dimensions of this upper chromosphere.

Preceding observations having shown that the light of the solar corona is composed for the most part of a small number of elementary rays differing considerably in refrangibility, it appeared to me that the form and dimensions of the higher chromosphere might be much more conveni:ntly studied by means of a large prism fixed in front of the object-glass of the telescope, whereby the several chromatic images of the corona would be distinctly formed in the focal plane. If the prism has but little dispersive power, and the eye-piece does not magnify too much, all the chromatic images of the corona may in this manner be observed simultaneously in the same field, and their form and dimensions directly investigated.

Towards the end of the year 1868, a small fint-glass prism was made for me by Signor Merz, of Monaco, to be fitted to the object-glass of the equatorial belonging to the Observatory of Campodoglio, for observations on the spectra of the stars; and this apparatus, in consequence of the dispersion of the prism, and the goodness of this prism and of the object-glass, was found to be admirably adapted for observing the eclipse in the manner just described.

The dispersion of the prism from the lines $\mathrm{C}$ to $\mathrm{H}$ of Fraunhofer is about $32^{\prime}$; the free aperture of the objectglass is $4 \frac{1}{3}$ French inches; the field of the telescope is about $x^{\circ}$, with a magnifying power of 40 .

My conviction of the great advantages which would be afforded by this instrument in the observation of the approaching eclipse, induced me to carry it to India for that purpose; and I was glad to learn that Mr. Lockyer, the chief of the expedition, had in like manner resolved to observe the corona by means of a spectroscope without a slit, being persuaded that this would be the most convenient method of solving the questions relating to the corona itself. With this instrument, then, I prepared to observe the eclipse, proposing to myself the following problems :--

vol. v,
I. To ascertain whether, just before the beginning, and at the end of totality, the solar spectral lines are reversed -a phenomenon observed by Prof. Young in the eclipse of 1870 .

2. Amidst the several chromatic images of the prominences, to observe especially whether the image given by the yellow line $D^{3}$ coincides with that of the lines of hydrogen gas.

3. To define the form and dimensions of the chromatic images of the corona.

The day before the eclipse, I delineated, by means of the direct-vision spectroscope applied to the telescope, the profile of the solar disc, in order to ascertain the state of the chromosphere at the several parts of the limb, and the protuberances existing there. But the picture did not come out with sufficient exactness, in consequence of the cloudy state of the sky, and the strong wind which prevailed throughout the day. This picture, however, clearly showed that both on the eastern and on the western limb, at the point where contact would take place between the lunar and solar discs in the total eclipse, the chromosphere was in that abnormal condition which is generally observed in the neighbourhood of solar spots.

The number of the prominences was, however, rather small, and their dimensions moderate; conditions which appeared to me to be favourable for the examination of the corona.

From the 5 th to the I th of December, the state of the sky at Poodocottah was somewhat variable; and generally, in the early hours of the day, great masses of mist and cloud predominated in the east, leaving but little hope in favour of our station for observing the eclipse. On the morning of the 12 th, indeed, the sky was almost wholly covered with dense masses of mist and cloud, completely obscuring the sun till $7 \mathrm{~h} .53 \mathrm{~m}$., at which time the eclipse had already begun. Soon after this the sun was again covered with thick clouds, but fortunately they began to break a few minutes before totality, when the bright disc of the sun was already sufficiently reduced, and when consequently the time for observation was rapidly approaching.

To verify the phenomenon of the reversal of the spectral lines at the extreme edge of the sun, I had arranged the plane of dispersion at right angles to the edge at the point of second contact.

At thirty seconds before totality, the spectral image of the luminous crescent was already sufficiently weakened to allow of its observation by the naked eye without a dark glass; and it was then that the principal dark lines of the solar spectrum came out distinct, and even more strongly marked than before, and curved parallel to the bright edge of the sun ; but a few seconds before totality these lines disappeared completely, and the spectrum became continuous, without however exhibiting, just before totality, the reversal of the lines, although I was watching most intently for this phenomenon. I would not, however, be understood as denying altogether the reversal of the lines, for it is not impossible that a thin film of mist, or the bright atmospheric light at that time diffused over the spectrum of the solar limb, may have concealed the bright lines.

At the very instant of totality, the field of the telescope exhibited a most astonishing spectacle. The chromo- 
sphere at the edge which was the last to be eclipsedsurmounted for a space of about $50^{\circ}$ by two groups of prominences, one on the right the other on the left, of the point of contact-was reproduced in the four spectral lines, $C, D^{3}, F$ and $G$, with extraordinary intensity of light and the most surprising contrast of the brightest colours, so that the four spectral images could be directly compared and their minutest differences easily made out.

In consequence of the achromatism of the objectglass, all these images were well defined, and projected in certain coloured zones, with the tints of the chromatic images of the corona. My attention was mainly directed to the comparison of the forms of the prominences on the four spectral lines, and I was able to determine that the fundamental form, the skeleton or trunk, and the principal branches, were faithiully reproduced or indicated in the images, their extent being, however, greatest in the red, and diminishing successively in the other colours down to the line $G$, on which the trunk alone was reproduced. In none of the prominences thus compared was I able to distinguish, in the yellow image $\mathrm{D}^{3}$, parts or branches not contatned in the red image $C$.

Meanwhile the coloured zones of the corona became continually more strongly marked, one in the red corresponding with the line $\mathrm{C}$, another in the green, probably coinciding with the line 1474 of Kirchhoff's scale, and a third in the blue perhaps coinciding with $F$.

The green zone surrounding the disc of the moon was the brightest, the most uniform, and the best defined. The red zone was also very distinct and well defined, while the blue zone was faint and indistinct. The green zone was well defined at the summit, though less bright than at the base; its form was sensibly circular and its height about $6^{\prime}$ or $7^{\prime}$. The red zone exhibited the same form and approximately the same height as the green, but its light was weaker and less uniform. The height of the green zone was estimated by comparison with the moon's diameter, and from the observed distance of the spectral lines of the prominences.

These coloured zones shone out upon a faintly illuminated ground, without any marked trace of colour. If the corona contained rays of any other kind, their intensity must have been so feeble that they were merged in the general illumination of the field.

Soon after the middle of the total eclipse, there appeared on the eastern limb, at about $110^{\circ}$ from the north point, a fine group of prominences formed of jets rather low but very bright, some rectilinear, others curved round the sun's limb, and exhibiting the intricate deviations and all the characters of prominences in the neighbourhood of solar spots. The brightness and colour of these $j \in t s$ were so vivid as to give them the appearance of fireworks.

The spaces between some of these jets were.perfectly dark, so that the red zone of the corona appeared to be entirely wanting there. Perhaps, however, this was only an effect of contrast due to the extraordinary brightness of the neighbouring jets. I have thought it right to refer to this peculiarity, because the appearance of interstices, or dark spaces, between prominences of considerable brightness, is often observed by means of the spectroscope, independently of total eclipses.

The want of an assistant to note the time, and to write down the observations as they were made, occasioned me some loss of time, and the end of the total eclipse was already at hand before I was aware of it.

The green and red zones were well developed at the western as at the eastern limb, while the blue remained faint and ill-defined. Soon afer the appearance of the chromosphere at the western edge, there was suddenly projected on the spectrum of the sun's limb, which then appeared beyond that of the moon, a stratum of bright lines, separated by dark spaces; but I could not deter. mine whether they were due to a general or partial reversal of the spectral solar lines, or to a simple discontinuity in the spectrum, since they were too soon immersed in a flood of light, which put an end to the totality of the eclipse.

About half an hour after the total eclipse, the sun vas obscured by clouds, so that I was unable to observe the end of the partial eclipse.

Later in the day, when the sky had become sufficiently clear, I observed with the spectroscope the state of the chromosphere, and of the protuberances existing upon it ; but in consequence of the cloudy state of the sky, the violent wind which prevailed, and the shortness of the time at my command, the picture was not sufficiently distinct and detailed.

L. RESPIGHI

\section{THE ZOOLOGICAL RECORD FOR I870}

The Zoological Record for 1870 ; being Vol. VII. of the "Record of Zoological Literature." Edited by Alfred Newton, F.R.S. (London: published by John Van Voorst, for the Zoological Record Association, 187I.) Pp. 523.

$7 \mathrm{HE}$ "Record of Zoological Literature" is already so well known to, and so well appreciated by, all students of zoology, that we need only remind our readers of the fact that, after five volumes had been published by Mr. Van Voorst, under the editorship of Dr. Günther, the publisher found it impossible to continue its publication, the actual yearly loss being something very considerable. It is true that the British Association for several years contributed $100 l$. towards this loss, and that three of the Recorders contributed, during the years that the British Association was so liberal, an equal sum out of their own pockets. Still, the expenses of such a work are so great, and the number of copies sold so small, that we were not surprised at Mr. Van Voorst's decision, nor to find that the present editor was compelled to look to the co-operation of zoologists generally to attain its continued publication; and it speaks much, not only for his energy, but also for the personal esteem with which he is regarded, that he could obtain in so short a time upwards of eighty friends who should guarantee $400 \%$. between them towards any loss that might accrue on this, the seventh volume. While we do not pretend to be in the councils of the committee of the Zoological Record Association, nor have we received even so much as a hint on the subject from the secretary, yet we may venture to express our belief that the members, while they will have the consciousness of having furthered the publication of this work, will not have to pay very much more for the seventh volume than they had for each of the previous six. 